\title{
Análise da resistência mecânica de vigas de mármore sintético através da teoria estatística de Weibull
}

\author{
Analysis of the strength of synthetic marble \\ beams through the statistical distribution of \\ Weibull
}

Ricardo Fouad Rabahi ${ }^{1}$, Flaminio Levy Neto ${ }^{2}$

\author{
${ }^{1}$ Universidade de Brasília - UnB/FT/ENM - CEP - 70910-900, Asa Norte, Brasília, DF \\ E-mail: ricardorabahi@gmail.com \\ ${ }^{2}$ Universidade de Brasília - UnB/FT/ENM - CEP - 70910-900, Asa Norte, Brasília, DF \\ E-mail: flaminio@unb.br
}

\begin{abstract}
RESUMO
No presente trabalho, adotou-se a análise estatística de Weibull para avaliação da resistência mecânica à flexão, e sua repetibilidade, de mármores sintéticos, tanto puros quanto reforçados com fibra vidro-E. O objetivo foi observar o comportamento do módulo de Weibull e da resistência mecânica, à medida que se introduz fibra de vidro picotada na composição do mármore sintético. Para isso foram avaliados quatro diferentes teores, em massa, de fibra de vidro, para estabelecer uma curva padrão do comportamento mecânico, à medida em que se alterou a fração de fibra do compósito. A metodologia de Weibull viabiliza esta análise, uma vez que, além dos corpos de prova possuírem grande semelhança dimensional de espessura com os produtos fabricados em mármore sintético, ela quantifica a ocorrência de imperfeições devido ao processo de fabricação. Os resultados demonstraram que a inserção de fibra de vidro, em uma massa de $3 \mathrm{Kg}$ de mármore sintético, em proporções de 12,5 a 100 gramas provocou, dependendo da quantidade de fibra inserida, uma variação na resistência intrínseca, que inicialmente diminuiu (0 a 25 g) e depois aumentou até o valor máximo com 100 gramas. Outro resultado importante foi o tempo de desmoldagem, que influenciou fortemente a dispersão dos resultados, que quando diminuído de $24 \mathrm{~h}$ para $2 \mathrm{~h}$, provocou uma diminuição do módulo de Weibull (i.e. aumento na variação da tensão de ruptura), de até 60\%.
\end{abstract}

Palavras chaves: Mármore Sintético, Fibra de Vidro, Estatística de Weibull.

\section{ABSTRACT}

In the present study, the Weibull statistical analysis was adopted for evaluating the flexural strength, as well as its repeatability, of cultured marbles, both pure and reinforced with fiberglass. The objective of this study was to observe the behavior of the Weibull modulus and mechanical strength, as chopped fiberglass was incorporated in the composition of cultured marble. For this purpose, four different compositions with different amounts of glass fiber, by weight, were evaluated, to establish a standard curve of the mechanical behavior of the composite fiber. The Weibull approach enables this analysis since, in addition to the dimensional thickness resemblance of test samples with the products made of synthetic marble, it quantifies the occurrence of imperfections due to the manufacturing process. The results showed that the glass fiber insertion into a mass of $3 \mathrm{~kg}$ of synthetic marble, in proportions of 12.5 to 100 grams, caused a variation in the intrinsic resistance, depending on the amount of fiber inserted, which initially fell ( 0 to25 g) and then increased to a maximum of 100 grams. Another important result was the time of demolding, which strongly influenced the dispersion of the results that, when reduced by $2 \mathrm{~h}$ to $24 \mathrm{~h}$, caused a decrease in Weibull modulus (i.e. increase in the variation of the rupture stress), up to $60 \%$.

Keywords: Synthetic Marble, Fiberglass, Weibull statistics.

\section{INTRODUÇÃO}

Em virtude da faixa de aplicações dos compósitos ser significativamente amplo, os requisitos de desempenho 
estrutural e baixo custo, aliados à cadência de produção apresentam diferentes graus de importância nos distintos segmentos industriais. Nas aplicações aeronáuticas, aeroespaciais e biomédicas o desempenho estrutural dos compósitos é de vital importância, ao passo que o fator econômico envolvido em sua utilização tem menor relevância. Na indústria automobilística e na construção civil, por outro lado, esta situação tende a ser inverter, ou seja, o baixo custo passa a ser um parâmetro preponderante [1]. Largamente utilizado na construção civil, o mármore sintético está longe de ser uma novidade no mercado, sendo sua aplicação mais usual pias, tanques, lavatórios e bancadas, com grande flexibilidade de tamanhos e formas, característica de seu processo de moldagem. Em particular, por alojarem menos bactérias que os mármores naturais, também vem sendo muito usados em hospitais e locais públicos [2]. Em função do aumento da competitividade, este tem se tornado um produto cada vez mais fino e leve, bem como com as mais variadas dimensões. Sem uma normatização especifica no Brasil, as empresas têm buscado, através do apelo do baixo custo, encontrar soluções para se manterem competitivas no mercado, seja buscando novas formas ou novas composições. Existem hoje no mercado de Mármore Sintético dois tipos principais de produto, o puro e o reforçado com fibra de vidro-E picada. Neste último, a fibra de vidro é adicionada posteriormente ao processo de moldagem com adição de uma camada de fibra picotada, juntamente com resina pura, na região inferior, menos visível e supostamente mais tracionada.

Apesar da falta de normatização no Brasil, já existem normas internacionais de avaliação, largamente utilizadas em trabalhos e artigos para pedras sintéticas como "Bowl Cracking in Sinks”, que consiste em aplicações cíclicas de choque por esferas e submetidas a uma variação de temperatura, provocando choques térmicos com agua corrente, para avaliação de sua vida útil, regidos por normas da ANSI (American National Standards Institute), ANSI Z 124.3 e ANSI Z 124.6, "Physical Testing of Castings”, que consiste em submeter a vários ensaios de resistência superficiais e estruturais regidos pela ASTM (American Society for Testing and Materials) como: "Barcol hardness" (D-2583) teste de dureza superficial, "Heat deflection temperature" (D-648) teste de deflexão com o aumento de temperatura, "Flexural strength and modulus” (D-790), ensaio de flexão para determinação da tensão de ruptura e módulo de elasticidade, “Tensile strength, modulus and elongation” (D-638) obtenção de módulo de elasticidade e alongamento, "Water absorption” (D-570) avalia a absorção de agua, [3]. Estes ensaios também são amplamente utilizados para caracterização das resinas termofixas, matéria prima básica para produção do mármore sintético, conforme podemos observar pela tabela de propriedades típicas do boletim técnico da REICHHOLD (tabela 1 [4]) e pela tabela de propriedades típicas boletim técnico da ARA QUíMICA (tabela 2 [5]).

Tabela 1: Propriedades tipicas do fabricante (Reichhold) da Resina Polylite ${ }^{\circledR}$ 32135-00 - Engineered Cast Marble Resin [4]

\begin{tabular}{|c|c|c|c|}
\hline \multicolumn{4}{|c|}{ PROPRIEDADES FÍSICAS TÍPICAS } \\
\hline PROPRIEDADES & UNIDADES & VALOR & MÉTODO DE TESTE \\
\hline Dureza Barcol & & 35 & ASTM D-2583 \\
\hline Temperatura de Distorção & ${ }^{\circ} \mathrm{C}$ & 53 & ASTM D-648 \\
\hline Resistência à Flexão & $\mathrm{MPa}$ & 103,42 & ASTM D-790 \\
\hline Módulo de Flexão & GPa & 4,137 & ASTM D-790 \\
\hline Resistência à Compressão & $\mathrm{MPa}$ & 96,53 & ASTM D-695 \\
\hline Resistência à Tração & MPa & 48,26 & ASTM D-638 \\
\hline Módulo de elasticidade & GPa & 3,792 & ASTM D-638 \\
\hline \% Alongamento na Ruptura & $\%$ & 1.5 & ASTM D-638 \\
\hline Absorção de Água 24 h. $25^{\circ} \mathrm{C}$ & $\%$ ganho de peso & 0.2 & ASTM D-570 \\
\hline Absorção de Água 2 h. $100^{\circ} \mathrm{C}$ & $\%$ ganho de peso & 1.0 & ASTM D-570 \\
\hline
\end{tabular}

O objetivo deste trabalho experimental, como forma de avaliar o desempenho mecânico de corpos de prova (CPs) de resina poliéster com carga de pó de mármore, é observar o comportamento do módulo de Weibull e da resistência mecânica intrínseca, à medida que se introduz fibra de vidro picotada na composição do mármore sintético. Sua influência na resistência intrínseca será investigada experimentalmente e levar-seá em consideração os ganhos ou perdas através de teste de flexão de três pontos conforme norma ASTM D790 [6]. Adicionalmente, também foi avaliada a repetibilidade dos ensaios, quantificada pelo Módulo de Weibull (m). Para isso foram avaliados quatro teores, em massa, de acréscimo de fibra de vidro, para estabelecer uma curva padrão que exponha o comportamento mecânico, à medida em que se altera o teor de fibra do compósito. 


\section{MATERIAIS E MÉTODOS}

\subsection{Considerações Gerais}

Os materiais empregados neste trabalho consistem, basicamente, de mármores sintéticos puros e conjugados com fibras picadas de vidro-E, em teores variados. Sendo que o mármore sintético é composto de resina poliéster insaturada ARAZYN 3.7 (nome comercial), carga mineral de carbonato de cálcio (CaCO3) e outros aditivos. A resina poliéster pura utilizada possui uma tensão de ruptura média, em ensaio de flexão, em torno de $82 \mathrm{MPa}$ (tabela 2), essa resistência pode variar significativamente conforme sua composição como podese observar em K. Raju et al, [3], com valores entre 93,5 MPa e 133,6 MPa. Ainda, em outro boletim do fabricante (REICHHOLD [5]) da resina POLYLITE® 32135-00 (tabela 1) com resistência à flexão de 103 MPa.

Tabela 2: Propriedades tipicas do fabricante (ARA QUÍMICA), Resina AZ3.7 [5]

Após adicionar-se o carbonato de cálcio, como carga de enchimento e diminuir custo, na proporção de $25 \%$ de resina poliéster e 75\% de carbonato de cálcio, por peso, a tensão de ruptura em flexão, varia entre 20 e 35

\begin{tabular}{|c|c|c|c|}
\hline \multicolumn{2}{|c|}{ PROPRIEDADES DA RESINA LÍQUIDA AZ 3.7 - Padrão } \\
\hline Propriedades & Unidades & Valores & Método de teste \\
\hline Temperatura de Distorção Térmica & ${ }^{\circ} \mathrm{C}$ & 75 & ASTM D-648 \\
\hline Resistência à Flexão & $\mathrm{MPa}$ & 82,74 & ASTM D-790 \\
\hline Módulo na Flexão & $\mathrm{GPa}$ & 2,48 & ASTM D-790 \\
\hline Resistência à Tração & $\mathrm{MPa}$ & 53,78 & ASTM D-638 \\
\hline Módulo na Tração & $\mathrm{GPa}$ & 1,34 & ASTM D-638 \\
\hline \% Alongamento & $\%$ & 3.0 & ASTM D-638 \\
\hline
\end{tabular}

MPa [2]. É de certa forma previsível que haja uma considerável perda de resistência na tensão de ruptura, uma vez que ocorre uma interferência da carga mineral cujas partículas atuam como concentradores de tensões. Em contrapartida, houve um ganho de espessura, consequentemente, no momento de inércia da área na secção transversal, isto, com um incremento no custo muito baixo (carbonato de cálcio).

A fibra de vidro-E é um reforço usual dos materiais compósitos poliméricos que proporciona, através do ancoramento de seus fios, em princípio, melhores propriedades mecânicas aos compósitos. Neste trabalho, a fibra acrescida no compósito é do tipo roving picotado por picotadeira pneumática, com comprimento médio de $50 \mathrm{~mm}$, e de aproximadamente 4000 fios por mexa. As amostras foram produzidas em moldes de fibra de vidro, especialmente confeccionados para este fim, na forma de cinco barras, com as seguintes dimensões de $8 \mathrm{~mm}$ x 40 mm x 1000 mm, posteriormente colocadas em uma mesa vibratória por 12 minutos, objetivando melhor compactação e para mitigar a formação de bolhas.

Existiu, porém, uma diferença básica entre os processos de confecção dos CPs, elaborados em 2 lotes. No primeiro lote, (grupos de amostras A, B e C), seguiu-se o padrão normal de desmoldagem do processo produtivo industrial, de 2 horas, que ocorre duas horas após a moldagem, posteriormente deixado um tempo de cura por 24 horas em grades de descanso. Já o segundo, (grupos de amostras D, E e F), a cura de 24 horas ocorreu com os CPs dentro dos moldes, não havendo nenhuma movimentação até sua cura final.

\subsection{CPs com cura fora dos moldes, desmoldados após 2 horas}

No processo de desmoldagem com 2 horas após a moldagem, os CPs possuem $1000 \mathrm{~mm}$ de comprimento por $40 \mathrm{~mm}$ de largura, sendo, portanto, peças esbeltas de pouca rigidez, antes da cura de 24 horas. Esse processo é normalmente utilizado no processo produtivo comum, para proporcionar maior utilização dos moldes no mesmo dia, em pias, tanques, lavatórios e outros produtos em mármore sintético. Normalmente, são peças de maiores espessuras nas bordas, que proporcionam maior rigidez, como também em muitos casos, possuem cubas que enrijecem a estrutura, não apresentando a mesma esbeltez dos corpos de prova analisados. As composições deste primeiro lote foram:

- Grupo A - 3,0 Kg de mármore sintético puro (i.e. sem fibras);

- Grupo B - 3,0 Kg de mármore sintético com 50 g de fibra de vidro; e

- Grupo C - 3,0 Kg de mármore sintético com 100 g de fibra de vidro. 
Foram realizados alguns ensaios preliminares para determinação de um percentual máximo de fibras, que permitisse à massa fluidez para moldagem e vibração. Essas misturas foram realizadas sem adição de catalizador, o que permitiu a inserção da fibra de forma gradual para que se estabelecesse os valores ideais para realização dos ensaios. Assim os grupos B e C foram acrescidos da quantidade média e máxima, respectivamente, sem que houvesse prejuízo no processo de moldagem quanto a fluidez da massa no molde. A partir deste resultado e baseado no estudo de VISION \& CHOU [14], passou se para análise do valor mínimo de incremento de fibras que proporcionasse ganhos na resistência à flexão realizando então, os ensaios $\mathrm{E}$ e F com valores inferiores à média.

\subsection{CPs desmoldados após 24 horas}

Ao término da moldagem e vibração das peças, os CPs deste segundo lote permanecem nos moldes por 24 horas até que já estejam com a cura completa tendo assim uma maior rigidez, como também uma maior facilidade de desmoldagem, provavelmente também, devido à maior contração da massa no interior do molde fêmea. As composições neste lote foram:

- $\quad$ Grupo D - 3,0 Kg de mármore sintético puro (i.e. sem fibras);

- Grupo E - 3,0 Kg de mármore sintético com 12,5 g de fibra de vidro; e

- Grupo F - 3,0 Kg de mármore sintético com 25 g de fibra de vidro.

\subsection{Ensaios de Flexão em Três Pontos}

O método de ensaio escolhido para determinação das propriedades mecânicas foi ensaio de flexão em três pontos, baseado na norma ASTM D-790-90 para compósitos (Figura 1). Os seis grupos de amostras, com no mínimo 25 CPs por composição, foram ensaiados em duas etapas, baseado na norma ASTM D-790-90 de flexão de materiais compósitos ([6], [16]), utilizando a máquina universal de ensaios MTS 810. Ao término da confecção das amostras obteve-se 25 amostras para os grupos A, B, e C, sendo que na desmoldagem realizada houve a perda de dois CPs em a cada grupo em função de um defeito no molde, que foi corrigido para os Grupos D, E e F que totalizou 27 amostras, todos eles, em média, com as seguintes dimensões nominais: $8 \mathrm{~mm}$ x 40mm x 140mm. A distância L entre os apoios é de $114 \mathrm{~mm}$

A flexão em três pontos é método indicado, pela simplicidade da confecção dos corpos de prova (CP), uma vez que existe uma maior dificuldade de se executar ensaios de tração em materiais frágeis, devido ao possível deslizamento do CP nas garras da máquina de ensaio e por melhor representar a condição real de fratura (em campo), Também é utilizado em vários artigos como ferramenta de análise, como podemos observar em XEIDAKIS et al. [7], que analisa vigas de mármore através da flexão de três pontos com materiais de composição diferentes, com o objetivo de compará-los por meio de seus desempenhos nos ensaios. Os fabricantes Reichhold e Ara Química, dentre outros, também adotam este método como forma de caracterizar seus produtos, conforme mostrado nas Tabela 1 e 2 respectivamente.

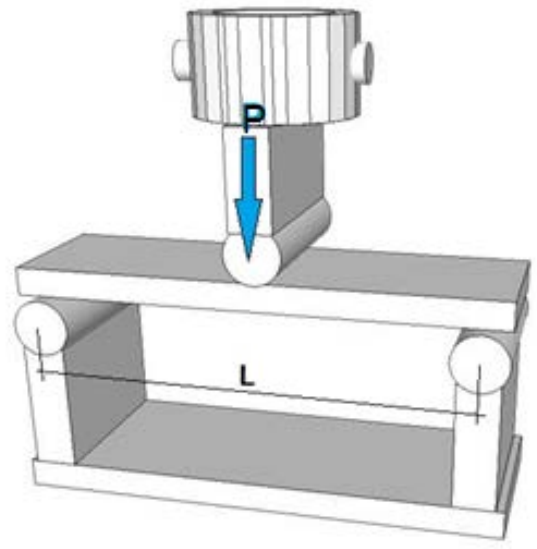

Figura 1: Ensaio de Flexão em três pontos

Ao se promover uma flexão aplicando uma força $P$ no centro do CP, conforme ilustrado na Figura 1, provoca-se, além da compressão na região superior, uma tensão de tração na inferior, que surge a partir espessura média e tendo maior valor na superfície inferior da amostra, onde, via de regra, terá início a fratura 
do CP. Basicamente, os valores fornecidos pelo equipamento durante o ensaio são: a carga aplicada $(P)$; o deslocamento vertical ou deflexão $(f)$ e o tempo em segundos. Em todos os ensaios destrutivos realizados, a velocidade da deflexão foi controlada e mantida no valor de $2 \mathrm{~mm} / \mathrm{min}$. A MTS-810 possui um sistema de aquisição de dados que registrou a força aplicada $(P)$, a deflexão $(f)$ e o tempo $(s)$.

\section{MODELAGEM TEÓRICA}

\subsection{Tensões Normais Máximas devido á Flexão}

A Tensão normal máxima devido á flexão (ou módulo de ruptura em flexão), $\sigma_{\max }$, obtido a partir do cálculo das tensões máximas de ruptura, que segundo NASH [13], e dado pela equação (1):

$$
\sigma=\frac{M_{f} * y}{I}
$$

Onde:

- $\quad \sigma$ é a tensão normal máxima devido à flexão;

- $\quad M_{f}$ é o momento fletor $(P / 2) *(L / 2)$;

- $\quad y=t / 2$ é a metade da altura da secção transversal do CP;

- $\quad$ I é o momento de inércia de área da secção transversal do CP.

- $\quad P$ é a carga aplicada

- $\quad L$ é a distância entre apoios

A partir da equação (1), chega-se então à equação (2), que descreve a Tensão máxima de ruptura em função das variáveis que podem ser obtidas através do experimento, quais sejam: a força $(P)$, a dimensões distância entre apoios $(L)$, espessura $(t)$, largura do CP $(b)$ e a deflexão $(f)$, esta última que pode ser utilizada para obtenção do módulo de elasticidade $\mathrm{E}$ do material. Observando que, via de regra, materiais dúcteis sofrem um dobramento significativo, e materiais frágeis fraturam mesmo com pequenas curvaturas,

$$
\sigma=\frac{M_{f} \frac{t}{2}}{\frac{b \cdot t^{3}}{12}}=\frac{\frac{P \cdot L}{4} \cdot \frac{t}{2}}{\frac{b . t^{3}}{12}}=\frac{3}{2} \frac{P \cdot L}{b \cdot t^{2}}
$$

\subsection{Estatística de Weibull}

Em função da grande variação dos resultados (fraturas frágeis), tornou-se imprescindível que os dados fornecidos pelo ensaio de flexão em três pontos tivessem um tratamento estatístico adequado. A análise proposta foi a de Weibull, pois ela descreve a fração das amostras que fraturam em diferentes tensões aplicadas. À medida que aumenta o teor de falhas e defeitos acumulados nos corpos de prova, diminui-se a tensão de ruptura, ficando caracterizada a analogia de que as correntes nunca são mais resistentes do que seu elo mais fraco, rompendo-se neste ponto. Assim, ao estender este conceito para os materiais frágeis, Weibull representou os elos por pequenos volumes da peça, sendo, o elo mais fraco, equivalente à região que contém a falha crítica. Weibull propôs também uma função empírica de distribuição de probabilidade de falhas e obteve a expressão para a probabilidade de fratura acumulada, conhecida como Distribuição de Weibull [8].

A distribuição de Weibull trabalha com a probabilidade do material, após a aplicação de uma determinada tensão, resistir ou não, mesmo que esta tensão esteja abaixo da média do limite de resistência estática convencional do material. Isto é, o rompimento do material se dará pela ocorrência de falhas em seu processo produtivo e a distribuição de Weibull quantifica esta probabilidade. Pode-se observar na Figura 2, a diferença de comportamento de um material dúctil e outro material frágil, na dispersão dos resultados, sendo o material dúctil de resultados menos dispersos [8].

O método, da posição relativa, utilizado para determinar as probabilidades de falha, que analisa um corpo de prova de volume $V$, poderá apresentar uma variação de falhas e descontinuidades que, estando ele submetido a uma tensão normal $\sigma$, pode-se subdividir em $n$ partes unitárias do sólido de volume $V_{0}$, sendo que cada elemento tem a mesma distribuição de defeitos, ou seja, a probabilidade de sobrevivência é $P\left(V_{0}\right)$ e vai ser igual à $1-F\left(V_{0}\right)$, onde $F\left(V_{0}\right)$ é probabilidade de falha [8]. Assim, inicialmente, ordena-se os resultados experimentais das tensões de falha de forma crescente (i.e. dá mais baixa à mais elevada), 
numerando-as a partir do número um até $n$ (número total de amostras) [9]. Assim, as probabilidades de falha $F(V) j$, em um lote

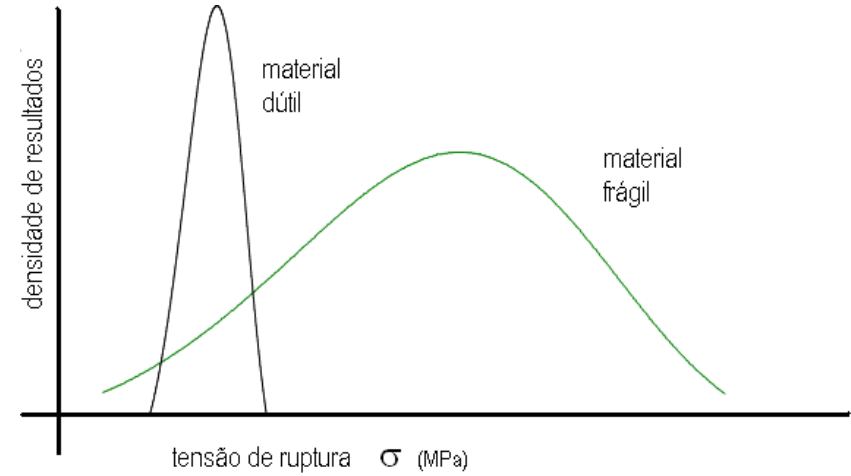

Figura 2: Distribuição de Weibull de amostras que fraturam sob tensão aplicada - material dúctil e material frágil, [8].

de $n$ CPs, para cada ensaio $j$, com $1 \leq j \leq n$, correspondem à classificação numérica (posição na tabela $(1,2, \ldots$, $n)$ ), dividido por $(n+1)$, ou seja, obtém-se $F(V)_{j}$, equação (3), para os valores de $\sigma_{\max }$, obtidos pela equação (2), que por sua vez, são os resultados experimentais dos ensaios e classificando-os então, pelo método da posição relativa [8].

$$
F(V)_{j}=\frac{j}{n+1}
$$

Para analisar um corpo de prova de volume $V$, que poderá apresentar uma variação de falhas e descontinuidades, estando ele submetido a uma tensão $\sigma$, pode-se subdividir em $n$ partes de volume $V_{0}$ onde cada parte tem a mesma distribuição de defeitos. Assim, segundo ASKELAND [8] "pode-se mostrar que a probabilidade de sobrevivência dos corpos de prova $P\left(V_{0}\right)$, ou seja, a probabilidade de um material frágil não frature sob a tensão aplicada $\sigma$ é dada pela equação (4).” Seguindo o mesmo raciocínio podemos ainda escrever que a probabilidade de falha $F\left(V_{0}\right)=1-P\left(V_{0}\right)$, e $P\left(V_{0}\right)$ é obtido da seguinte equação:

$$
P\left(V_{0}\right)=\exp \left[-\left(\frac{\sigma-\sigma_{u}}{\sigma_{0}}\right)^{m}\right]
$$

A partir da equação (4), onde $\sigma$ é a tensão aplicada, $\sigma_{0}$ a resistência intrínseca que correspondente a probabilidade de sobrevivência de $37 \%$ ou ainda $P\left(V_{0}\right)=0,37$, e $\sigma_{u}$ é a tensão onde a probabilidade de falha é nula, ou ainda, a probabilidade de sobrevivência é 1,0 . O módulo de Weibull $m$ pode variar de 0 a aproximadamente 100 e representa a repetibilidade da resistência mecânica do material, esses valores estão diretamente ligados às características do material analisado como, homogeneidade, quantidade de defeitos como bolhas, impurezas ou descontinuidades, entre outros. O módulo de Weibull $m$ tem valores próximos a 3 para cerâmicas (materiais frágeis) e próximo a 100 para metais e ligas (dúcteis). Alguns materiais cerâmicos especiais podem alcançar um módulo $m$ entre 5 e 10 [8].

Para materiais frágeis onde existe uma variação considerável nos valores correspondentes às tensões de ruptura, adota-se como valor seguro, quando não haverá falha no material testado, a tensão de ruptura onde a probabilidade de falha é nula, $\sigma_{u}$, igual a 0 , assim para esses materiais pode-se através da equação (3) encontrar a seguinte equação:

$$
F\left(V_{0}\right)=1-P\left(V_{0}\right)=1-e \quad \square\left[-\left(\frac{\sigma}{\sigma_{0}}\right)^{m}\right]
$$

Portanto, analisando a equação (5), pode-se constatar que para uma tensão aplicada $\sigma$ nula, a probabilidade de sobrevivência, com certeza, será de $100 \%$ e ainda com o acréscimo da tensão aplicada $\sigma$, a probabilidade de sobrevivência $P\left(V_{0}\right)$ diminui, alcançando valores próximo a zero para tensões aplicadas $\sigma$, muito maiores que a resistência intrínseca $\sigma_{0}$. Assim, a partir também da equação (3) pode-se comprovar que quando $\sigma=\sigma_{0}$ a probabilidade de sobrevivência $P\left(V_{0}\right)$ passa a ser igual a $1 / e \approx 0,37$ ou $37 \%$, ou ainda que a probabilidade de falha $F\left(V_{0}\right)$ seja aproximadamente igual a 0,63 , ou $63 \%$ [8].

O expoente $m$, chamado de Módulo de Weibull, é adimensional e fornece uma avaliação da 
homogeneidade do material e, consequentemente, da repetibilidade de seus valores de resistência e de sua confiabilidade estrutural. Quanto menor o módulo de Weibull, maior é a dispersão dos resultados, tornando o material susceptível a falhas em uma maior faixa de tensões. Já para valores elevados do parâmetro, a dispersão torna-se menor em um estreito intervalo em torno de $\sigma_{0}$. Assim, quanto maior o $m$, maior é a confiabilidade do material, pois a dispersão das tensões de ruptura medidas é menor.

Após desenvolvimento da equação (5), o Modulo de Weibull pode ser determinado, para materiais frágeis, pela equação (6):

$$
m=\frac{\ln \left(\ln \left(\frac{1}{1-F(V)}\right)\right)}{\left(\ln (\sigma)-\ln \left(\sigma_{0}\right)\right)}
$$

Existem outras formas de se avaliar a probabilidade, porém segundo BERGMAN [12] o método proposto por WILKS [9], apresenta menor variância.

Obtém-se assim a seguinte equação para o cálculo de m em um intervalo de resultados AB:

$$
m=\frac{\ln \left(\ln \left(\frac{1}{1-F(V) a}\right)\right)-\ln \left(\ln \left(\frac{1}{1-F(V) b}\right)\right)}{\left(\ln \left(\sigma_{a}\right)-\ln \left(\sigma_{b}\right)\right)}
$$

Sendo plotado um gráfico onde a ordenada seja dada pela expressão $\ln \left[\ln \left(1 / 1-F\left(V_{0}\right)\right]\right.$ e a abscissa por $\ln \left(\sigma / \sigma_{0}\right)$, pode-se concluir que o módulo m representa a inclinação de uma reta interpolada a partir dos pontos obtidos neste gráfico, conforme ilustrado na figura 3 [8], [10] e [11], onde, $F(V)$ é obtido pelo método da posição relativa, $\sigma_{a}$ e $\sigma_{b}$ obtidos pela equação (4) e m é representado pela inclinação da reta definida pelos pontos $\mathrm{AB}$, como mostra a figura 3 .

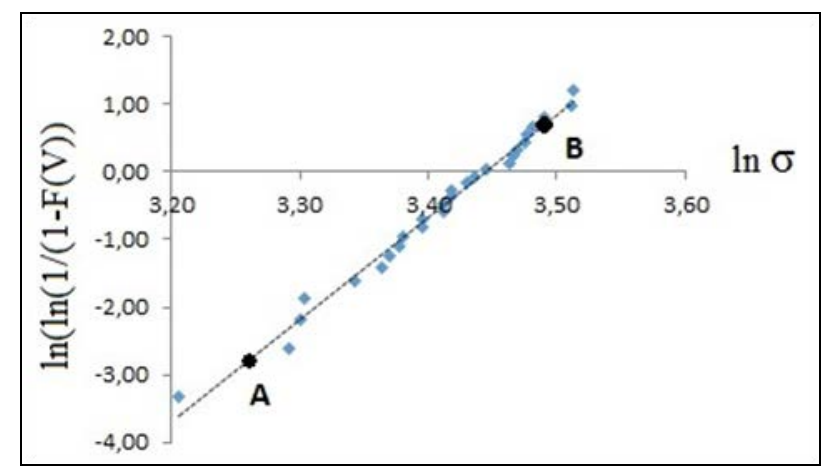

Figura 3: Exemplo de gráfico de $\ln (\ln (1 /(1-F(V)) \times \ln \sigma)$ [17]

\section{RESULTADOS}

A metodologia de Weibull viabiliza esta análise, uma vez que, além dos corpos de prova possuírem grande semelhança dimensional de espessura com os produtos fabricados comercialmente em mármore sintético, eles estão sujeitos à ocorrência de imperfeições devido ao processo de fabricação. A inserção de fibra de vidro na massa de mármore sintético, em proporções de 12,5 a 100 gramas, provoca uma diminuição no módulo de Weibull, de até 25\%; e, dependendo da quantidade de fibra inserida a resistência intrínseco pode aumentar ou diminuir, atingindo o valor mínimo com 25 gramas. O tempo de desmoldagem, também influencia fortemente o módulo de Weibull. Os resultados encontrados foram:

- $\quad$ Primeiro lote (grupos a, b e c) de ensaios realizado com desmoldagem duas horas após a moldagem

- Grupo “A”: $m=8,09, \sigma_{0}=29,63 \mathrm{MPa}, \sigma_{\text {max.medio }}=29,76 \mathrm{MPa}$

- Grupo "B”: $m=6,08, \sigma_{0}=31,61 \mathrm{MPa}, \sigma_{\text {max.medio }}=32,17 \mathrm{MPa}$

- Grupo “C”: $m=6,03, \sigma_{0}=35,01 \mathrm{MPa}, \sigma_{\text {max.medio }}=35,58 \mathrm{MPa}$

- Segundo lote (grupos d, e e f) de ensaios realizado com desmoldagem 24 horas após a moldagem.

- Grupo “D”: $m=16,57, \sigma_{0}=32,52 \mathrm{MPa}, \sigma_{\text {max.medio }}=32,26 \mathrm{MPa}$ 
- Grupo "E": $m=15,04, \sigma_{0}=30,17 \mathrm{MPa}, \sigma_{\text {max.medio }}=30,06 \mathrm{MPa}$

- Grupo "F": $m=14,05, \sigma_{0}=28,73 \mathrm{MPa}, \sigma_{\text {max.medio }}=28,54 \mathrm{Mpa}$

Tabela 3: Resultados de Resistências Intrínsecas $\left(\sigma_{0}\right)$ e Módulos de Weibull $(m)$

\begin{tabular}{|c|c|c|c|c|c|c|}
\hline & Grupo A & Grupo B & Grupo C & Grupo D & Grupo E & Grupo F \\
\cline { 2 - 7 } & \multicolumn{2}{|c|}{ DESMOLDADOS COM 2 } & \multicolumn{3}{|c|}{ DESMOLDADOS COM 24 } \\
HORAS & HORAS \\
\hline$m$ & 8,09 & 6,08 & 6,03 & 16,57 & 15,04 & 14,05 \\
\hline$\sigma_{0}(\mathrm{MPa})$ & 29,63 & 31,61 & 35,01 & 32,52 & 30,17 & 28,73 \\
\hline $\begin{array}{c}\text { Quantidade de } \\
\text { fibra de vidro (g) }\end{array}$ & 0 & 50,0 & 100 & 0 & 12,5 & 25 \\
\hline
\end{tabular}

Objetivando criar uma referência para a análise da influência do acréscimo de fibra, entre $0 \mathrm{~g}$ e $100 \mathrm{~g}$ de fibra de vidro, unificou-se os resultados, para elaboração da tabela 4, onde adotou-se um valor médio entre os grupos "A" e "D", levando-se em consideração que a obtenção dos resultados a partir destes grupos, teve como diretriz a manutenção de todos os parâmetros de produção dos CPs, com exceção dos tempos de desmoldagem que foram diferentes.

Tabela 4: Resultados de Resistências Intrínsecas $\left(\sigma_{0}\right)$ em ordem crescente de acréscimo de Fibra de Vidro

\begin{tabular}{|c|c|c|c|c|c|}
\hline & $\begin{array}{c}\text { Média dos } \\
\text { Grupos A e D }\end{array}$ & Grupo E & Grupo F & Grupo B & Grupo C \\
\hline $\begin{array}{c}\text { Quantidade de } \\
\text { fibra de vidro (g) }\end{array}$ & 0 & 12,5 & 25 & 50 & 100 \\
\hline$\sigma_{0}(\mathrm{MPa})$ & 31,08 & 30,17 & 28,73 & 31,61 & 35,01 \\
\hline$m$ & 12,33 & 15,04 & 14,05 & 6,08 & 6,03 \\
\hline$E(\mathrm{GPa})$ & 7,76 & 9,61 & 8,09 & 7,68 & 6,7 \\
\hline
\end{tabular}

\section{DISCUSSÃO}

O decréscimo inicial na resistência intrínseca, dos grupos E e F, com a inserção de fibra de vidro, de certa forma não era o esperado, pois a resistência mecânica da fibra de vidro é muitas vezes maior que a resistência do mármore sintético. Isso deveria provocar uma melhora em suas propriedades mecânicas. Porém, pode-se suspeitar que as fibras inseridas na massa, abaixo da fração critica [14], possam ter incrementado as falhas e/ou bolhas na massa de mármore sintético, uma vez que para se ter eficiência de atuação das fibras na massa é necessário que haja uma perfeita adesão das mesmas, e que em quantidades inferiores a $50 \mathrm{~g}$ por $3 \mathrm{~kg}$ de massa inviabiliza o aumento da tensão de ruptura, provocando inicialmente diminuição em sua resistência.

Na figura 4, obtida a partir tabela 4, mostra-se um diagrama da resistência intrínseca média dos grupos de CPs, em função dos teores de fibra de vidro picadas, adicionado em cada grupo, obtendo uma resistência intrínseca $\sigma_{0}$ mínima com acréscimo de $25 \mathrm{~g}$ de fibra de vidro em $3 \mathrm{Kg}$ de massa de mármore sintético. Notase que o Grupo C apresenta $\sigma_{0} 18,2 \%$ superior ao Grupo A (mesmo lote de ensaio) e $13 \%$ superior em relação à média dos Grupos $\mathrm{A}$ e $\mathrm{D}$ (Grupos sem adição de fibra de vidro). 


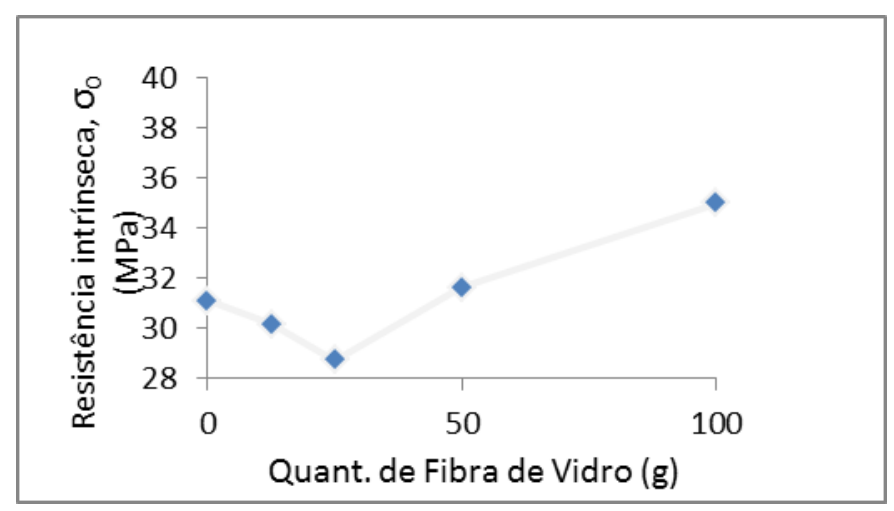

Figura 4: Comportamento da Resistência Intrínseca [MPa] x Quant. de Fibra de Vidro [g]

Como no mármore sintético ensaiado neste trabalho, as fibras são picotadas e aleatórias, a chance de elas virem a atuar como defeitos, quando inseridas em quantidade insuficiente, é bem maior que no caso de reforço contínuo e unidirecional, na direção de carregamento de tração uniaxial, que gerou o gráfico mostrado na figura 5 [14].

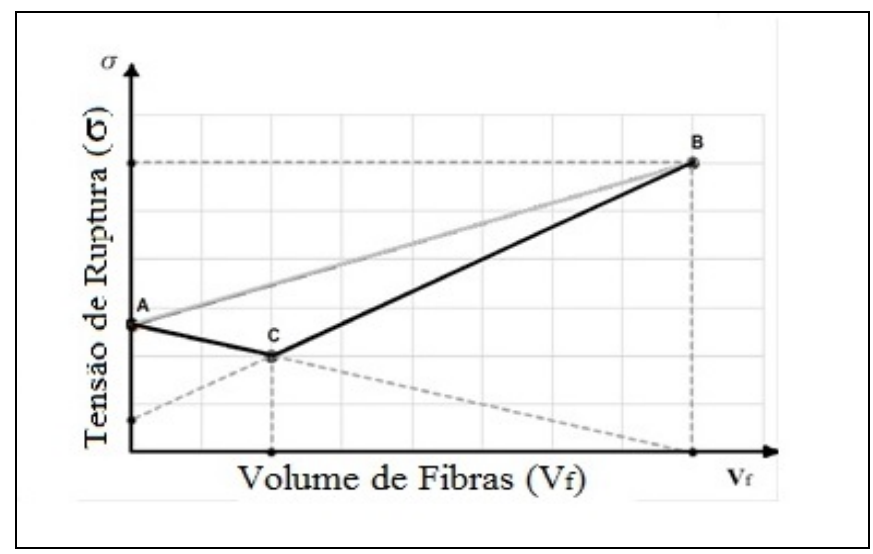

Figura 5: Diagrama da influência de diferentes frações volumétricas de fibras adaptado de VINSON \& CHOU [14].

Por se tratar de dois lotes de amostras (A, B e C), (D, E e F) distintos, porém com formulação de massa de mármore sintético, idênticas, percebe-se que ao iniciar-se o acréscimo de fibra de vidro, até 25g, ocorre uma diminuição na resistência intrínseca do material, que pode ser atribuída a uma atuação negativa do material fibroso na composição, há indicações de que a matriz de mármore sintético predomina na resistência do compósito e as fibras atuam como se fossem defeitos [14].

Em seguida, com acréscimos maiores esse comportamento se inverte a partir de $50 \mathrm{~g}$ de fibra de vidro, ou aumentando ainda mais com $100 \mathrm{~g}$ de fibra para $3 \mathrm{~kg}$ de massa. Ou seja, as fibras de vidro só passam a atuar como reforço a partir de uma fração volumétrica mínima, ou fração volumétrica critica.

Com relação ao Módulo de Weibull, $m$, observa-se uma ligeira tendência de queda nas duas etapas, um pouco mais acentuada no lote de CPs desmoldados após 2 horas (Grupos A, B e C), na medida em que se acrescenta a fibra de vidro. Tendência essa que se mostra menos intensa no lote de CPs desmoldados após 24 horas (Grupos D, E e F), e quando se passa de $50 \mathrm{~g}$, com $m=6,45$, e para $100 \mathrm{~g} m=6,35$, indicando assim uma estabilização no valor do módulo de Weibull $(m)$.

A grande diferença entre os valores de $m$ entre lotes de CPs: (i) desmoldados após 2 horas (Grupos A, B e C), com média de 32,08 MPa; e (ii) desmoldados após 24 horas (Grupos D, E e F), com média de 30,47 $\mathrm{MPa}$, pode ser atribuída ao melhor controle na segunda etapa, no processo de elaboração dos corpos de prova. Por exemplo, na segunda etapa os corpos de prova foram desmoldados no dia seguinte, ou seja, após 24 horas; e na primeira etapa os corpos de prova foram desmoldados 2 (duas) horas após a moldagem. Esse tempo de duas horas é o normalmente empregado dentro do processo produtivo industrial, visando melhor aproveitamento dos moldes. Porém, para que haja esta desmoldagem somente duas horas após, é necessário que haja um cuidado especial, pois os CPs saem da fôrma com pouca rigidez; o que pode, em alguns casos, provocar micro trincas ocorrendo assim uma instabilidade a mais nos valores obtidos. Provavelmente causado por um número maior de defeitos gerados na operação de desmoldagem, e, uma possível ocorrência 
de micro trincas explicaria o fato dos Módulos de Weibull $(m)$ dos CPs desmoldados após 2 horas terem apresentado valores de $m$, em média, menores, por um fator superior a duas vezes, em relação aos desmoldados após 24 horas.

Já nos grupos D, E e F, a desmoldagem após 24 horas permitiu que a cura da matriz polimérica se efetivasse totalmente e o compósito ficasse bem mais rígido e melhor consolidado antes de ser sacado dos moldes. O aumento significativo no módulo de Weibull dos CPs dos grupos D, E e F, em relação aos dos grupos $\mathrm{A}, \mathrm{B}$ e $\mathrm{C}$, de cerca de duas vezes (i.e. fator de $\approx 2$ ), sugere que no lote referente aos grupos $\mathrm{D}, \mathrm{E}$ e $\mathrm{F}$, desmoldados após 24 horas, a qualidade do produto final é bem melhor, havendo menor quantidade de defeitos, em relação aos grupos A, B e C, desmoldados com 2 horas, antes da matriz polimérica atingir a máxima rigidez. Vale lembrar que a geometria dos corpos de prova é bem esbelta sem nervuras, concavidades ou sobressaltos, diferindo assim das peças normalmente inseridas no processo produtivo industrial.

O decréscimo na resistência intrínseca com a inserção de fibra de vidro, de certa forma não era o esperado, pois, a resistência mecânica da fibra de vidro é muitas vezes maior que a resistência do mármore sintético, e, isso deveria provocar uma melhora em suas propriedades mecânicas, porém pode-se suspeitar que as fibras inseridas na massa possam ter incrementado as falhas e/ou bolhas na massa de mármore sintético, uma vez que para se ter eficiência de atuação das fibras na massa é necessário que haja um perfeito engastamento ou adesão das mesmas, que em quantidades inferiores a $50 \mathrm{~g}$ por $3 \mathrm{~kg}$ de massa inviabiliza o procedimento provocando diminuição em sua resistência.

É interessante considerar ainda, que a aplicação de reforço na massa de mármore sintético possa incrementar o processo produtivo da pedra sintética, auferindo a ela um ganho ainda maior na utilização em processo de produção por extrusão, onde a proporção de inserção de fibras poderá alcançar patamares superiores aos $100 \mathrm{~g}$ por $3 \mathrm{~kg}$ de massa, obtidos neste trabalho, já que o processo por extrusão aufere uma maior capacidade de molhamento das fibras e cargas através da pressão aplicada pelo processo, permitindo assim uma menor quantidade relativa de resina na massa total [15].

\section{CONCLUSÕES}

Em síntese, as principais conclusões foram:

Em relação à média dos valores dos grupos sem fibras de vidro picadas (A e D), de $\sigma_{0}=32,32 \mathrm{MPa}$, a adição de fibras, até 25 g, causou uma progressiva diminuição na Resistência Intrínseca $\left(\sigma_{0}\right)$ para 28,73 MPa. Sendo que com 12,5 g de fibras obteve-se o valor intermediário $\sigma_{0}=30,17 \mathrm{MPa}$;

Para a incorporação de 50 g de fibra de vidro, a Resistência Intrínseca atingiu um valor de 31,61 MPa, próximo ao valor inicial de 29,63 com aumento de 6,7\%. E, ao se adicionar $100 \mathrm{~g}$ de fibras, $\sigma_{0}$ subiu para 35,01 MPa (valor máximo), um aumento de 18,2\%;

O aumento no tempo de desmoldagem, de duas para 24 horas, fez o Módulo de Weibull, $m$, aumentar por um fator superior a duas vezes. Ou seja, os CPs desmoldados após 24 apresentaram uma repetibilidade na resistência mecânica bastante superior;

O Módulo de Weibull $(m)$ médio dos CPs desmoldados após 2 horas foi de 6,73. Nestes grupos, A, B e C, $m$ diminuiu de 8,09 para 6,08 e depois 6,03, quando os teores de fibra foram, respectivamente, 0,50 e 100 g; e o Módulo de Weibull $(m)$ médio dos CPs desmoldados após 24 horas foi de 15,22. Nestes grupos, D, E e F, $m$ diminuiu de 16,57 para 15,04 e depois 14,05, quando os teores de fibra foram, respectivamente, 0 , 12,5 e 25 g.

A adição de fibra de vidro em massa de mármore sintético pode, portanto, representar um ganho significativo na resistência à flexão, de até 18,2\%, desde que observados critérios mínimos de adição, bem como tempo de desmoldagem, o qual influencia fortemente na dispersão dos resultados obtidos, sendo que, na desmoldagem com 24 horas obteve-se menor variação dos resultados que na desmoldagem com 2 horas, da ordem de 60\% menor, avaliado pela dispersão média entre os lotes A, B, C e D, E, F.

\section{AGRADECIMENTOS}

Ao Programa de Pós-Graduação de Ciências Mecânicas, PCMEC, da Universidade de Brasília pela oportunidade oferecida na realização deste Mestrado, à Petrobrás, e a todos que direta ou indiretamente contribuíram para conclusão deste trabalho. 


\section{BIBLIOGRAFIA}

[1] LEVY NETO, F., PARDINI, L.C., Compósitos Estruturais: Ciência e Tecnologia, 1 ed, Editora Edgard Blucher, São Paulo, 2008.

[2] RABAHI, R.F., DINOAH, J., GUEDES, J.,et al. - Estudo da Influência da Fibra de Vidro nas Propriedades Mecânicas do Mármore Sintético, Relatório de Curso na Pós-Graduação, PCMEC - UnB, Brasília, 2007.

[3] RAJU, K., LEMING, C., OWEN, G. “A New Class of Resins tor Superior Thermal Shock Resistance in Composite Sinks”, 46th Annual Conference, Composites Institute, The Society of the Plastics Industry, Inc. February 18-21, 1991.

[4] REICHHOLD, Product Bulletin - Polylite 32135-00 Engineered Cast Marble Resin, Reichhold, Research Park, NC, USA, 2008.

[5] ARA QUÍMICA S/A, Arazyn 3.7 Resina Poliéster Insaturado, Orto-Tereftálica, Média Reatividade Boletim Técnico, Ara Química, Araçariguama - SP, 2007.

[6] ASTM, D-790-10- Standard Test Methods for Flexural Properties of Unreinforced and Reinforced Plastics and Electrical Insulating Materials, West Conshohocken, PA 19428-2959, United State, EDT 2010.

[7] XEIDAKIS, G.S., SAMARAS, I. S., ZACHAROPOULOS, D. A., et al., "Crack-Growth In A MixedMode Loading On Marble Beams Under 3 Point Bending", International journal of fracture, 79(2), pp. 197208, 1996.

[8] ASKELAND, D.R., PHULÉ, P.P., Ciência e Engenharia dos Materiais, CENGAGE Learning, pp. 190196, Rio de Janeiro, 2008.

[9] WILKS, S.S., “Determination of Sample Size for Setting Limits”, The Annals of Mathematical Statistics, n.12, p.91-96, 1942.

[10] GREEN, D. An Introduction to the Mechanical Properties of Ceramics, Cambridge University Press, pp. 286-290, Cambridge, 1998.

[11] MATTHEUWS, F. L., RAWLINGS, R. D. Composite Materials: Engineering and Science. Imperial College of Science, Technology and Medicine, London, UK, 1994.

[12] BERGMAN, B. “On the Estimation of the Weibull Modulus”, Journal of Material Science Letters, n.3, p.689-692, 1983.

[13] NASH, W., Resistência dos Materiais, Mac Gral Hill, São Paulo, 1992.

[14] VINSON JACK, R. AND T.-W. CHOU. Composite materials and their use in structures., Applied Science Publishers Ltd, London,1975.

[15] CAUHOIS, J.P. Compósitos 1 - Materiais, Processos, Aplicações, Desempenhos e Tendências, ABMACO - Associação Brasileira de Materiais Compósitos, Cap. 07, 2012

[16] WHITNEY, J. M., DANIEL, I. M., PIPES, R. B. Experimental Mechanics of Fiber Reinforced Composite Materials, The Society for Experimental Mechanics, Brookfield Center, Connecticut, New Jersey, USA, 2000

[17] RABAHI, R.F. Análise da Resistência de Vigas De Mármore Sintético Através da Distribuição Estatística de Weibull, Tese de M.Sc Mestrado, Universidade de Brasília, Departamento de Engenharia Mecânica, 2010. 